\title{
Editorial for the Special Issue of EJKM 2021
}

For close to 60 years, knowledge management has been emerging, evolving and adapting as a discipline and an area of practice. Over the decades, the field has drawn from many and diverse fields, including economics, education, computer science, business administration, communication, information science, and sociology.

Throughout these years, the heart of the knowledge management domain has been knowledge and people. However, as digital transformation efforts have gradually become both a priority and a critical management issue for businesses of all types, the digital workspace increasingly supports key knowledge management functions such as team collaboration, improved workflows and the sharing and reuse of knowledge assets.

On the 11 March 2020, the World Health Organization declared the COVID-19 outbreak as a pandemic. Our work environment shifted dramatically, and remote work meant that productivity, collaboration and culture were dramatically affected. At that point, those trends that were emerging prior to 2020 accelerated to define our new world. The initial concern of surviving the pandemic turned into unprecedented levels of collaborations and exchange of knowledge between organisations across the globe as they sought to innovate. New knowledge was created to understand and fight the virus. Organisational learning allowed for engineering companies and even universities to design and manufacture personal protective equipment to support the health sector. Knowledge was shared across sectors, as transport manufacturers and companies that normally produced medical devices collaborated for the production of ventilators to treat COVID-19 patients. Throughout this process, knowledge management gained importance as an enabler of our work, performance and innovation.

For organisations that chose -or were forced, to work remotely, knowledge management provided improved mechanisms to collaborate and share resources, capture the knowledge from experts who retired or change jobs as a result of the pandemic, and even establish new connections and a culture of learning. Today, knowledge management programs have become enterprise-wide and cross-corporate initiatives, increasingly central to any forward-looking business strategy.

Whilst still in the midst of a worldwide pandemic when this special issue was moving to publication, the debate in many organisations has shifted to the feasibility of the full return to the office and the extent to which technology-enabled remote and hybrid work will become the norm. This scenario would require a new level of maturity for knowledge management strategies and tools, so that the discipline continues to enable long-term performance as we transition from an industrial to a knowledge economy.

To achieve this, knowledge management scholars and practitioners will need to remain focused on challenges that pre-dated the pandemic, such as the development and improvement of knowledge management programs, as well as metrics that allow for quantification of their business value. A better understanding of its impact on the bottom line will help increase participation, engagement and support for knowledge management. The ways traditional in-person experiences are transferred to a virtual environment to enable knowledge flows in a work environment heavily dominated by technologies will also require further attention. While significant progress has been made since the start of 2020, the impact of remote and hybrid work on the effectiveness of knowledge management is only now beginning to be understood. There is a need to study how this new context enables, for example, collaboration across organisational boundaries. Monitoring and surveillance of employees working remotely have also increased, which may have a negative effect of knowledge sharing and adoption.

However, our constantly changing environment has also highlighted the need for the knowledge management discipline -enabled by the right technology, to expand its support to most business priorities. Take Artificial Intelligence (AI) as an example. While its principles are being widely adopted, many organisations have found themselves unable to make Al work for them. In theory, Al can help with automation of processes such as knowledge capture and harvesting, supporting knowledge search and discovery strategies, and ultimately driving better financial and operational performance. However, in order for Al-and technologies in general, to deliver on their promises, a solid knowledge management foundation is required. For example, contents that would drive decisions should be accurate and up to date; competencies that would drive collaborations should be accurately described and quantified; different repositories should be aligned in their content classification strategies. 
Knowledge management is also key to the digital resilience of the business. For example, in responding to the cyberattack that affected Norsk Hydro -one of the world's largest aluminium producers in 2019, the company had to overcome operational, threat-related, and technological knowledge barriers. A key theme throughout its response was that the company was able to effectively identify, capture and use necessary knowledge both within and outside of its boundaries. Examples of this ranged from its ability to regain otherwise lost know-how (from retirees and ex-employees), to the effective involvement of law enforcement and industry partners as sources of technical and procedural know-how.

In addition to increased productivity and the sustainable digital transformation of the enterprise, knowledge management can have a positive impact on strategic and operational areas including the quality of products and services; the organisation's ability to innovate; customer and employee experience; continuous learning at individual and organisational levels in the digital workspace; and even diversity and inclusion in the workplace. However, its success in achieving this impact requires the knowledge management discipline to continue to integrate both with information technologies and with the strategic goals of the organisation through key management functions such as risk management and value management.

This special issue was developed to bring together the experience of knowledge management scholars and practitioners on the challenges and opportunities of the discipline in the current socio-economic context. These articles cover subjects ranging from communities of practice to digital transformation, ethics and human behaviour, all of which determine the way the knowledge management discipline can rise to the stature of other business functions and operations. It is hoped that the issue will contribute to the current debate on how knowledge management can align with, and support the way the organisation works today and in the future.

\title{
Guest Editor
}

Alexeis Garcia-Perez, Centre for Business in Society, Coventry University, UK

\author{
Supported by \\ Anitha Chinnaswamy, Aston Business School, Aston University, UK \\ Vahid Jafari-Sadeghi, Aston Business School, Aston University, UK
}

